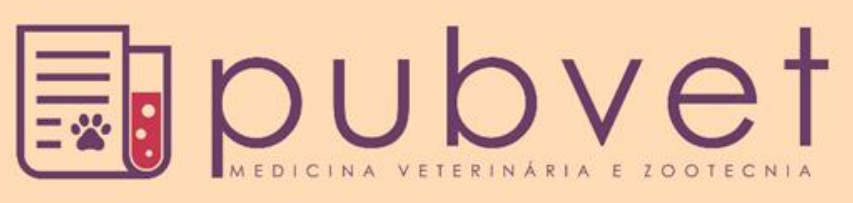

ISSN $1982-1263$

https://doi.org/10.31533/pubvet.v13n6a341.1-5

\title{
Eficácia do uso de Ivermectina e Praziquantel associados, em equinos da raça Crioula
}

Julia Somavilla Lignon $^{1 *}$, Tainá Ança Evaristo ${ }^{1}$, Alice Mueller $^{1}$, Aline Bianca Bervian $^{1}$,

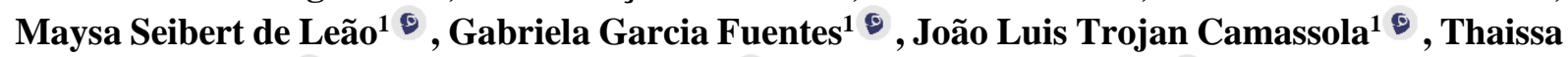
Gomes Pellegrin $^{1}{ }^{\bullet}$, Felipe Geraldo Pappen ${ }^{2} \theta$, Diego Moscarelli Pinto ${ }^{2} \bullet$

${ }^{I}$ Aluno(a) da Graduação da Universidade Federal de Pelotas, Departamento de Medicina Veterinária. Pelotas-RS, Brasil. ${ }^{2}$ Professor Doutor da Universidade Federal de Pelotas, Departamento de Medicina Veterinária Preventiva. Pelotas-RS, Brasil.

*Autor para correspondência, E-mail: julialignon@gmail.com

Resumo. O estudo teve como objetivo avaliar a eficácia anti-helmíntica da associação dos princípios ativos Ivermectina e Praziquantel, em equinos da raça Crioula, naturalmente parasitados. Foram utilizadas fezes de 68 animais, adultos, de ambos os sexos, criados em sistema extensivo e naturalmente infectados por helmintos. As amostras foram processadas e analisadas através da técnica de Gordon \& Whitlock (1939) e a avaliação da eficácia da associação dos princípios ativos foi baseada no método de Coles et al. (1992). O Índice de Eficácia do tratamento com a associação de Ivermectina e Praziquantel foi de 93,2\%. Assim pode-se concluir que a associação dos princípios ativos utilizados, foi eficaz no tratamento das endoparasitas na população de equinos estudada.

Palavras chave: anti-helmíntico, equinocultura, OPG, parasitos gastrintestinais

\section{Efficacy of the combination of Ivermectin and Praziquantel in Criollo horses}

\begin{abstract}
The objective of this study was to evaluate the anthelmintic efficacy of the combination of the active principles Ivermectin and Praziquantel in naturally occurring Criollo horses. Feces were used of 68 adult animals of both sexes, raised in an extensive system and naturally infected with helminths. Samples were processed and analyzed by the technique of Gordon \& Whitlock (1939) and the evaluation of the effectiveness of the association of active principles was based on the method of Coles et al. (1992). The Efficacy Index of treatment with the combination of Ivermectin and Praziquantel was $93,2 \%$. Thus, it can be concluded that the association of the active principles used was effective in the treatment of endoparasite in the studied equine population.
\end{abstract}

Keywords: antihelmintic, echinoculture, OPG, gastrointestinal parasites

\section{Eficacia de la asociación de Ivermectina y Praziquantel en equinos de la raza Criolla}

Resumen. El estudio tuvo como objetivo evaluar la eficacia antihelmíntica de la asociación de los principios activos Ivermectina y Praziquantel, en equinos de la raza Criolla, naturalmente parasitados. Se utilizaron heces de 68 animales, adultos, de ambos sexos, criados en sistema extensivo y naturalmente infectados por helmintos. Las muestras fueron procesadas y analizadas a través de la técnica de Gordon \& Whitlock (1939) y la evaluación de la eficacia de la asociación de los principios activos se basó en el método de Coles et al. 
(1992). El Índice de eficacia del tratamiento con la asociación de Ivermectina y Praziquantel fue del 93,2\%. Así se puede concluir que la asociación de los principios activos utilizados fue eficaz en el tratamiento de los endoparásitos en la población de equinos estudiada.

Palabras clave: antihelmíntico, equinocultura, OPG, parásitos gastrointestinales

\section{Introdução}

A equinocultura é uma atividade relevante na economia brasileira. O país possui animais de alto valor zootécnico que agregam importantes valores financeiros. Segundo o MAPA (2016), o setor teve grande evolução nos últimos anos, atingindo $\mathrm{R} \$ 16,15$ bilhões. Além destes aspectos, no Rio Grande do Sul, os costumes vinculados às tradições gaúchas trazem à tona uma maior importância a criação de cavalos, somando-se sua importância cultural, no trabalho, esporte e lazer.

Entre as patologias que afetam a sanidade dos equinos, as endoparasitoses são importantes causas de morbidade e mortalidade (Andrade et al., 2009; Botelho et al., 2012). O manejo extensivo, aliado ao excesso de animais nos piquetes, aumenta a propensão ao parasitismo (Reinemeyer, 1986; Reinemeyer \& Nielsen, 2013). Possíveis consequências vão desde redução da digestão e absorção de nutrientes, podendo levar a ruptura intestinal e morte. Contudo, alguns animais poderão apenas manifestar doença subclínica (Cutolo et al., 2011).

O controle parasitológico é principalmente com anti-helmínticos, cuja administração deve reduzir a eliminação de ovos nas fezes e a contaminação ambiental (Stratford et al., 2011). Essa forma de controle é adotada pela facilidade de aplicação, compra e custo. Todavia, com a rápida disseminação da resistência anti-helmíntica, por uso indiscriminado, essa medida tem sido vista como insustentável (Molento et al., 2008). Para o controle parasitário eficiente, medidas estratégicas e efetivas devem ser realizadas com o intuito de avaliar a espécie parasitária prevalente na região ou propriedade, para que seja escolhido o medicamento de melhor eficácia e assim conter a intensidade da infecção (Reinemeyer, 2012).

Uma das formas de melhor utilização dos compostos antiparasitários é a combinação de drogas, dificultando o aparecimento de genes da resistência (Molento et al., 2008). Em vista disso, o presente estudo teve como objetivo avaliar a eficácia anti-helmíntica da associação dos princípios ativos Ivermectina e Praziquantel, em equinos da raça Crioula.

\section{Material e métodos}

O experimento foi realizado em um criatório de equinos da raça Crioula em Camaquã no estado do Rio Grande do Sul (RS), Brasil, entre os meses de novembro e dezembro de 2018. Foram utilizadas fezes de 68 animais, adultos, de ambos os sexos, criados em sistema extensivo e naturalmente infectados por helmintos. O estudo foi aprovado pelo Comitê de Ética em Experimentação Animal da Universidade Federal de Pelotas (UFPel) sob o registro 7888.

As coletas foram realizadas préviamente ao tratamento com Invermectina $(0,2 \mathrm{mg} / \mathrm{kg})$ associada ao Praziquantel $(2,5 \mathrm{mg} / \mathrm{kg})$, formulado comercialmente, e uma segunda coleta foi feita transcorridos 14 dias após o tratamento. As amostras de fezes dos animais foram retiradas diretamente da ampola retal com auxílio de luvas e sacos plásticos. Foram identificadas individualmente, acondicionadas em recipientes isotérmicos com gelo reutilizável e encaminhadas ao laboratório do Grupo de Estudos em Enfermidades Parasitárias (GEEP) da Faculdade de Veterinária (FaVet) da Universidade Federal de Pelotas (UFPel), onde foram processadas e analisadas pela técnica de Gordon \& Whitlock (1939) sendo o resultado dado em ovos por grama de fezes (OPG) pré e pós-tratamento.

A avaliação da eficácia da associação dos princípios ativos foi baseada no método de Coles et al. (1992), o qual avalia a redução na contagem de ovos de helmintos por grama de fezes (OPG) no pré e pós tratamento.

Os resultados obtidos foram submetidos a análises descritivas e, posteriormente, a inferência estatística, sendo estabelecidas médias amostrais para o grupo de pré-tratamento e de pós-tratamento

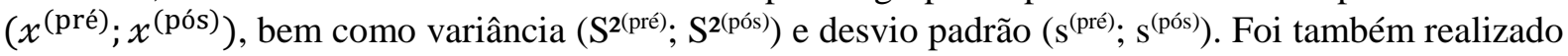


o Intervalo de Confiança (IC) para ambas médias amostrais $\left(\mu^{1} ; \mu^{2}\right)$, seguindo Distribuição Normal Padrão (Z), com nível de significância de $5 \%(\alpha=1,96)$, sendo $\mu^{1}=$ pré-tratamento e $\mu^{2}=$ póstratamento.

Conforme a configuração dos dados utilizou-se o Teste de hipótese unilateral a direita para médias, com nível de significância de 5\%, em Distribuição Normal Padrão (Z; $n \geq 30$ ).

\section{Resultados e discussão}

Nos exames fecais foi observada a infecção moderada e alta por parasitos da superfamília Strongylidea, e em menores graus, respectivamente, por Strongyloides spp., Oxyuris spp., e Parascaris spp., os quais foram identificados pela morfologia do ovo segundo Hoffmann (1987).

Os resultados do teste da redução da contagem de ovos por grama de fezes (TRCOF) de parasitos gastrintestinais, pela associação dos princípios ativos Ivermectina e Praziquantel estão expressos na tabela 1. Este método utilizado é o mais adequado para avaliar a eficácia de todas as classes de medicamentos disponíveis no mercado (Kaplan \& Nielsen, 2010; Von Samson-Himmelstjerna, 2012).

Tabela 1. Valores médios de OPG pré e pós-tratamento e Índice de Eficácia do tratamento com a associação de Ivermectina e Praziquantel

\begin{tabular}{lccc}
\hline Número de animais & Média de OPG pré-tratamento & Média de OPG pós-tratamento & Índice de Eficácia (\%) \\
\hline 68 & 1.694 & 114 & $93,2 \%$ \\
\hline
\end{tabular}

De modo geral, consideram-se como critérios para a suspeita de resistência, resultados inferiores a 95\% e com limite inferior do intervalo de confiança abaixo de 90\% (Coles et al., 1992). Kaplan \& Nielsen (2010) sugeriram o estabelecimento de valores mínimos específicos para cada classe de medicamentos, sendo: $90 \%$ para benzimidazóis, $90 \%$ para pirantel e $95 \%$ para as lactonas macrocíclicas.

Estabeleceu-se, com base nos valores de média, variância e desvio padrão, o Intervalo de Confiança com 5\% de significância (IC: $\alpha=1,96$ ) para ocorrência de ovos de helmintos gastrointestinais nesta população de equinos da raça Crioula. Desta forma, o resultado obtido em pré-tratamento antihelmíntico foi $I C\left(\mu^{1}\right)=\left(1.524,34 \leq \mu^{\text {(pré) }} \leq 1.836,66\right.$; e $=169,66$ ovos/helmintos $)$ e, em póstratamento anti-helmíntico foi $I C\left(\mu^{2}\right)=\left(0,32 \leq \mu^{(p o ́ s)} \leq 227,38 ; \mathrm{e}=113,68\right.$ ovos/helmintos) (Tabela 2).

Tabela 2. Média, variância e desvio padrão da prevalência de ovos de helmintos gastrointestinais em equinos da raça crioula: pré-tratamento e pós-tratamento com associação de Ivermectina e Praziquantel.

\begin{tabular}{lcc}
\hline & Pré-tratamento & Pós-tratamento \\
\hline$x$ (n\%ovos) & 1.694 & 114 \\
$\mathrm{~S}^{2}$ (n\%ovos) & 1.956 .281 & 231.779 \\
$\mathrm{~S}$ (n\%ovos) & 1.399 & 481 \\
\hline
\end{tabular}

Legenda: $x$ - média amostral em número de ovos; $\mathrm{S}^{2}$ - variância amostral em número de ovos; $\mathrm{S}$ - desvio padrão amostral em número de ovos.

Para o Teste de Hipóteses para médias amostrais, comparando as médias de ocorrência de ovos de helmintos gastrointestinais em equinos da raça Crioula tendo pré-tratamento e pós-tratamento antihelmíntico da associação de Ivermectina e Praziquantel, optou-se pela lateralização do teste a direta, com significância de 5\% $(\alpha=+2,58)$, tendo maior seletividade ao resultado, onde Ho: $\mu^{1}=\mu^{2}$ e Ha: $\mu^{1}>\mu^{2}$.

Foi aceito Ha, seguindo Distribuição Normal Padrão $(\mathrm{Z})$, com Z calculado $=+2,785$ e Z tabelado $=$ $+2,58$, em Teste de Hipótese Unilateral à Direita, conforme o que foi pressuposto na metodologia admite-se que estatisticamente há maior taxa de ovos de helmintos no resultado das médias no momento de pré-tratamento, quando comparado ao pós-tratamento antiparasitário, rejeitando-se Ho, admitindo-se Ha, ao nível de significância de 5\%. 


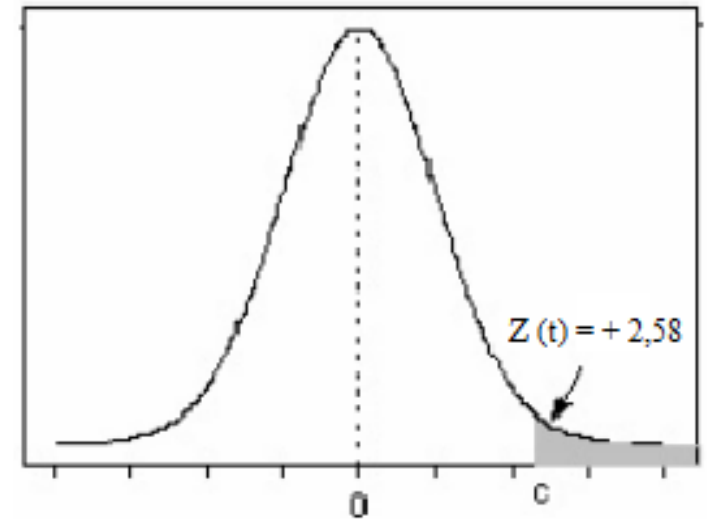

Figura 1. Demonstração gráfica da aplicação referida no Teste de Hipótese Unilateral a Direita para comparação entre duas médias amostrais entre a ocorrência de ovos de helmintos gastrointestinais em dois grupos de equinos da raça Crioula, tendo $\alpha=5 \%$, seguindo Distribuição Normal Padrão (Z). Legenda: $\mathrm{Z}(\mathrm{t})$ - valor crítico de $\mathrm{Z}$ tabelado, determinante para rejeição da Hipótese de Nulidade (Ho).

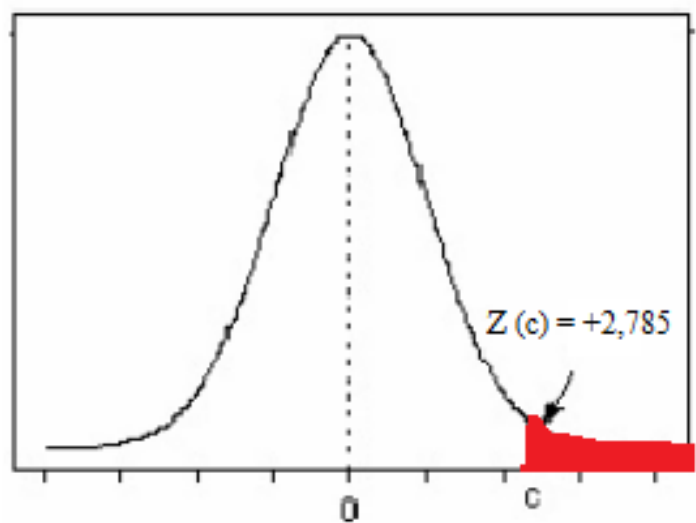

Figura 2. Demonstração gráfica do resultado da aplicação referida ao Teste de Hipótese Unilateral a Direta para comparação entre duas médias amostrais entre a ocorrência de ovos de helmintos gastrointestinais em dois grupos de equinos da raça Crioula, rejeitando-se $\mathrm{Ho}$, admitindo-se $\mathrm{Ha}$, havendo redução estatística nos índices parasitários obtidos em pós-tratamento anti-helmíntico com a associação de Ivermectina e Praziquantel. Legenda: Z (c) - valor crítico de $\mathrm{Z}$ calculado, determinando rejeição em Ho, admitindo-se Ha.

De acordo com Hipótese de Nulidade (Ho): admite-se que não há diferença estatística entre a taxa de ocorrência de helmintos gastrointestinais entre o pré-tratamento e pós-tratamento desta referida população de equinos da raça Crioula; Hipótese Alternativa (Ha): admite-se que há maior taxa de ocorrência de helmintos gastrointestinais nos equinos desta população amostral antes de serem tratados com a associação anti-endoparasitária Ivermectina e Praziquantel.

Dobrowolski et al. (2016) encontraram o valor de índice de eficácia de 99,09\% do produto contra ciatostomíneos no município de Guarapuava no Paraná. Felippelli et al. (2015) determinaram a eficácia deste anti-helmíntico no grupo de equinos infectados por Strongylus vulgaris, Trichostrongylus axei, Triodontophorus serratus e os ciatostomíneos, observando porcentagem de $100 \%$ em $T$. serratus e $S$. vulgaris; $99,98 \%$ em ciatostomíneos e 90,88\% em T. axei.

Assim, é ressalta-se a importância da realização de exames coprológicos para o monitoramento da eficácia dos princípios ativos em cada propriedade, pois é imprescindível conhecer a espécie prevalente dos parasitos e dessa forma realizar a escolha correta do fármaco a ser utilizado. Além disso, aliado ao diagnóstico e tratamento químico devem estar associadas medidas preventivas de manejo para retardar o aparecimento da resistência dos parasitos frente aos antiparasitários (Molento, 2005). Segundo Reinemeyer \& Nielsen (2013), a eficácia dos anti-helmínticos deve ser monitorada, no mínimo anualmente, em todos equinos da propriedade, ou numa amostra representativa no caso de grandes populações.

\section{Conclusão}

A associação dos princípios ativos utilizados, Ivermectina e Praziquantel, foi eficaz no tratamento das espécies de endoparasitas na população de equinos estudada.

\section{Referências bibliográficas}

Andrade, R. L. F. S., Carvalho Sobral, J. \& Silva, K. M. G. (2009). Avaliação clínica, hematológica e parasitária em equinos de tração na cidade de Aracajú, Sergipe. Acta Veterinaria Brasilica, 3(3):138142.

Botelho, G. G., Cassiano, A. d. L. V., Botelho, C. F. M. \& Botelho, C. M. (2012). Análise hematológica, bioquímico-sérica e coproparasitológica de equinos criados em Seropédica, RJ. Brazilian Journal of Veterinary Medicine, 34(1):69-72. 
Coles, G. C., Bauer, C., Borgsteede, F. H. M., Geerts, S., Klei, T. R., Taylor, M. A. \& Waller, P. J. (1992). World Association for the Advancement of Veterinary Parasitology (WAAVP) methods for the detection of anthelmintic resistance in nematodes of veterinary importance. Veterinary Parasitology, 44(1-2):35-44.

Cutolo, A. A., Santos, A. T. d. \& Allegretti, S. M. (2011). Field study on the efficacy of an oral 2\% ivermectin formulation in horses. Revista Brasileira de Parasitologia Veterinária, 20(2):172-175.

Dobrowolski, E. C., Slompo, D., Carrasco, A. d. O. T. \& Seki, M. C. (2016). Eficácia do praziquantel e da ivermectina em equinos infectados naturalmente com ciatostomíneos. Revista Acadêmica: Ciência Animal, 1475-81.

Felippelli, G., Cruz, B. C., Gomes, L. V. C., Lopes, W. D. Z., Teixeira, W. F. P., Maciel, W. G., . . . Soares, V. E. (2015). Susceptibility of helminth species from horses against different chemical compounds in Brazil. Veterinary Parasitology, 212(3-4):232-238.

Gordon, H. M. \& Whitlock, H. V. (1939). A new technique for counting nematode eggs in sheep faeces. Journal of the Council for Scientific and Industrial Research, 12(1):50-52.

Hoffmann, R. P. (1987). Diagnóstico de parasitismo veterinário. Porto Alegre, Brasil: Sulina.

Kaplan, R. M. \& Nielsen, M. K. (2010). An evidence-based approach to equine parasite control: It ain't the 60s anymore. Equine Veterinary Education, 22(6):306-316.

MAPA - Ministério da Agricultura, Pecuária e Abastecimento (2016). Revisão do Estudo do Complexo do Agronegócio do Cavalo. Disponível em: $\lfloor$ http://www.agricultura.gov.br/assuntos/camarassetoriais-tematicas/documentos/camaras-setoriais/equideocultura/anos-anteriores/revisao-doestudo-do-complexo-do-agronegocio-do-cavalo/view>.Acesso em: 10 março de 2018.

Molento, M. B. (2005). Resistência parasitária em helmintos de equídeos e propostas de manejo. Ciência Rural, 35(6):1469-1477.

Molento, M. B., Antunes, J., Bentes, R. N. \& Coles, G. C. (2008). Anthelmintic resistant nematodes in Brazilian horses. Veterinary Record, 162384-385.

Reinemeyer, C. R. (1986). Small strongyles. Recent advances. The Veterinary clinics of North America. Equine practice, 2(2):281-312.

Reinemeyer, C. R. (2012). Anthelmintic resistance in non-strongylid parasites of horses. Veterinary Parasitology, 185(1):9-15.

Reinemeyer, C. R. \& Nielsen, M. K. (2013). Handbook of equine parasite control. Iowa, USA: WileyBlackwell.

Stratford, C. H., McGorum, B. C., Pickles, K. J. \& Matthews, J. B. (2011). An update on cyathostomins: anthelmintic resistance and diagnostic tools. Equine Veterinary Journal, 43133-139.

von Samson-Himmelstjerna, G. (2012). Anthelmintic resistance in equine parasites-detection, potential clinical relevance and implications for control. Veterinary Parasitology, 185(1):2-8.

Recebido: 15 de março, 2019.

Aprovado: 30 de abril, 2019.

Publicado: 13 de junho, 2019.

Licenciamento: Este artigo é publicado na modalidade Acesso Aberto sob a licença Creative Commons Atribuição 4.0 (CCBY 4.0), a qual permite uso irrestrito, distribuição, reprodução em qualquer meio, desde que o autor e a fonte sejam devidamente creditados. 